\title{
Float-polishing process and analysis of float-polished quartz
}

\author{
S. F. Soares, D. R. Baselt, J. P. Black, K. C. Jungling, and W. K. Stowell
}

\begin{abstract}
A fluid-mechanical model is developed for the float-polishing process. In this model laminar flow between the sample and the lap results in pressure gradients at the grooves that support the sample on a fluid layer. The laminar fluid motion also produces supersmooth, damage-free surfaces. Quartz substrates for applications in high-stress environments were float polished, and their surfaces were analyzed by optical scatterometry, photoacoustic spectroscopy, and atomic force microscopy. The removal of $100 \mu \mathrm{m}$ of material by a lapping-polishing process, with final float polishing, left low levels of subsurface damage, with a surface roughness of approximately $0.2-\mathrm{nm}$ rms.
\end{abstract}

Key words: Float polishing, quartz, surface and subsurface characterization.

\section{Introduction}

Float polishing is an ultrafine polishing technique for the fabrication of optical, electronic, and magnetic components with damage-free surfaces. ${ }^{1}$ In float polishing a weighted sample (1-2 kg) and the lap are submerged in a slurry composed of deionized water and a measure of polishing powder. The sample and the lap are rotated in the same circular direction, usually at the same rate. Under equilibrium conditions the sample floats on a fluid layer above the lap and is simultaneously bombarded by microscopic polishing particles in the slurry. The apparatus for float polishing is shown in Fig. 1. The lap is mounted on a precision hydraulic bearing, and the runout at the periphery of the rotating plate is a $1-\mu \mathrm{m}$ total indicator reading, and the end play is also approximately $1 \mu \mathrm{m}$. Sn, $\mathrm{Cu}$, and nylon are typical lap materials, and the polishing face is machined in situ with natural diamond tools to a precision finish.

When this research was done S. F. Soares, J. P. Black, and K. C. Jungling were with the Center for the High Technology Materials, University of New Mexico, Albuquerque, New Mexico 87131, D. R. Baselt was with the Department of Chemical Physics, 127-72, California Institute of Technology, Pasadena, California 91125, and W. K. Stowell was with Phillips Laboratory, Kirtland Air Force Base, Albuquerque, New Mexico 87185. S. Soares is now with the Jet Propulsion Laboratory, California Institute of Technology, M.S. 238-528, Pasadena, California 91109. J. P. Black is also with Rockwell International, Kirtland Air Force Base, Albuquerque, New Mexico 87185.

Received 18 May 1992; revised manuscript received 19 March 1993.

0003-6935/94/010089-07\$06.00/0.

(C) 1994 Optical Society of America.
The groove pattern is usually a combination of spirals with the dimensions shown in Fig. 1. The sample and lap can be rotated at rates of as high as $200 \mathrm{rpm}$ in our system.

The thickness of the fluid layer between the sample and the lap is of the order of a micrometer, ${ }^{2}$ while the polishing particle sizes range from $1 \mathrm{~nm}$ (colloidal $\left.\mathrm{SiO}_{2}\right)$ to $100 \mathrm{~nm}\left(\mathrm{TiO}_{2}, \mathrm{Al}_{2} \mathrm{O}_{3}, \mathrm{CeO}_{2}\right.$, and $\left.\mathrm{MgO}\right)$. The particle size may be chosen to be larger than the fluid-layer thickness in which case mild abrasive action results. This may be the case with a $\mathrm{TiO}_{2}$ slurry in which the polishing particles initially conglomerate in the slurry but eventually break down to a finer size, resulting in a change in the consistency of the slurry. The slurry temperature in final float polishing is controlled by recirculation through a heat exchanger, and the polishing environment is enclosed and maintained at the class 100 cleanliness level.

The removal mechanism by which the sample is polished in a slurry could be kinetic, chemical, or by a diffusion process. ${ }^{3}$ The removal rate in float polishing is a function of several variables, some of which are the polishing powder, the sample, and the lap materials, the $\mathrm{pH}$ and the temperature of the slurry, the groove pattern, the rotational rates of the sample and the lap, and the sample loading.

Float polishing is also reputed to be an essentially damage-free polishing process. ${ }^{1}$ The system parameters such as the rotational rates of the sample and the lap and the groove profile and pattern on the lap allow laminar fluid flow in the system. We show by a simple model that, when the flow between the sample and the lap is laminar, the floating process is a result of pressure gradients at the lap grooves. We also 


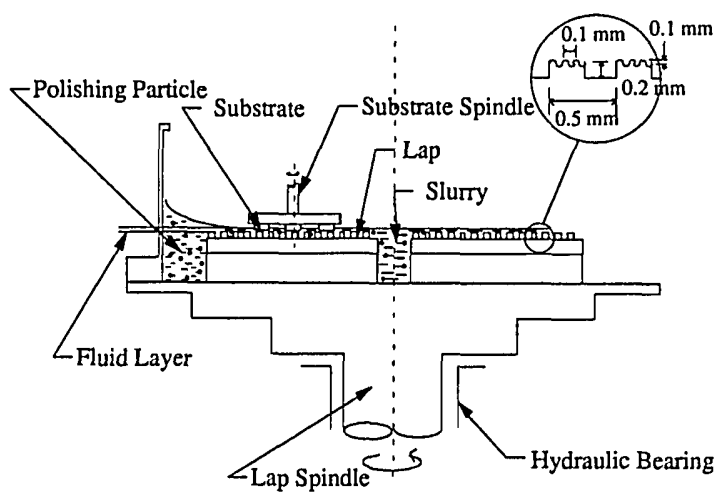

Fig. 1. Schematic of the apparatus for float polishing. The groove pattern consists of two superimposed diamond-turned spirals. The groove profile is shown in the inset.

show experimentally that quartz samples polished in this laminar system had a high-quality surface finish and low levels of subsurface damage. These damagefree quartz substrates have potential for use as oscillators in high-stress environments.

\section{Fluid-Mechanical Processes in Float Polishing}

The Reynolds numbers for the fluid flows between the sample and the lap and the motion of a polishing particle in the slurry are well below the critical value. The fluid motion in float polishing is thus laminar, and, on a microscopic level, float polishing falls into the category of a Couette fluid flow (see Fig. 2). ${ }^{4}$ This flow occurs between two parallel planes, one of which may be in motion relative to the other. One can understand the floating mechanism by making an analogy with another Couette system: the inclinedslipper bearing. In this system a planar load is supported by a fluid layer on an infinite plane in motion. 4,5 The load is slightly tilted in the direction of motion of the plane. If a laminar flow is assumed, a positive pressure gradient exists in the direction of
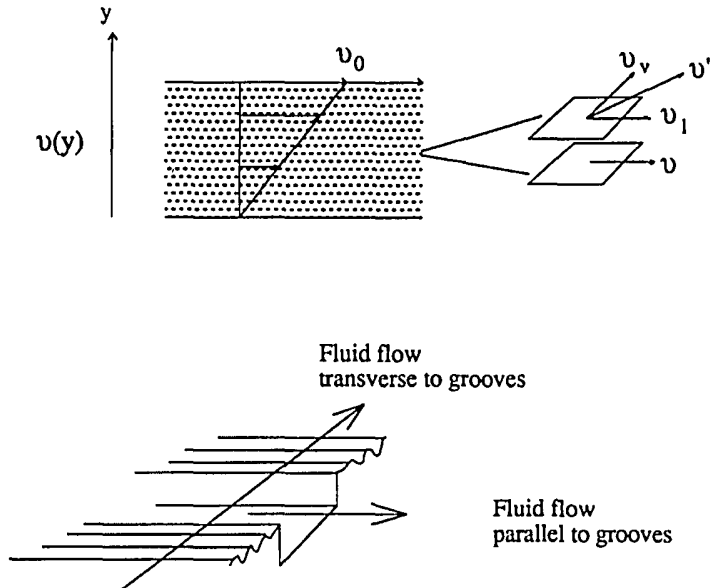

Fig. 2. Fluid motion in float polishing. The fluid motion at consecutive laminae is shown. $\nu_{1}$ and $\nu_{\mathrm{v}}$ are the laminar and vortex components of the lamina with velocity $\nu^{\prime}$ relative to the lower lamina with velocity $\nu, \quad \nu_{0}$ is the velocity of the upper plane relative to the lower plane. motion. The gradient is zero near the edge and is negative beyond the edge at which the pressure reaches its ambient value. Under these conditions the load is sustained by the fluid layer below it.

An alternative method of supporting a load is to substitute a corrugated infinite sheet for the infinite plane. The load need not be inclined in this system. The corrugation period must be less than the dimension of the load as shown in Fig. 3. The pressure gradients are also shown in the figure, and the load is supported at the points of high pressure. In comparison with the slipper bearing, the thickness of the fluid layer could be a function of the groove profile, the flow rate, and the fluid viscosity. ${ }^{5}$ In both of the above situations it is important that any inertial forces be negligible in comparison with the viscous forces existing in the fluid to maintain laminar flow. This is essentially the case in float polishing in which the lap is rotated at a slow rate, and the flow of the slurry across the lap grooves provides the pressure gradients that are necessary to support the samples on a layer of polishing fluid.

A simple one-dimensional model assists in understanding some of the parameters that are involved in this floating process. In this model we attempt to show only the feasibility of the floating process. A complete solution to the float-polishing process involves a complex-numerical three-dimensional analysis of laminar and vortical flows across superimposed groove patterns with complex boundary conditions and is not attempted here. We assume that unidirectional, laminar Couette flow exists between two parallel planes, one of which has a small quadratic boss in its profile. The details are shown in Fig. 4 in which the geometry is inverted as opposed to float polishing to simplify the calculation. There is also the possibility of a pressure gradient in the $y$ orientation; however it is not obvious that this component affects the floating process. If we assume hydrodynamic equilibrium, the forces acting on a differential fluid element are

$$
p \mathrm{~d} y \mathrm{~d} z-(p+\mathrm{d} p) \mathrm{d} y \mathrm{~d} z+(\tau+\mathrm{d} \tau) \mathrm{d} x \mathrm{~d} z-\tau \mathrm{d} x \mathrm{~d} z=0,
$$

where $p$ is the local pressure, $\tau$, the shear stress between two adjacent laminae, is given by Newton's law: $\tau=\eta(\mathrm{d} v / \mathrm{d} y)$, and $\nu$ is the local velocity.

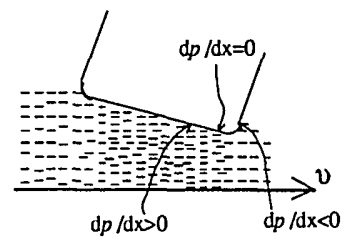

Inclined Slipper Bearing

(a)

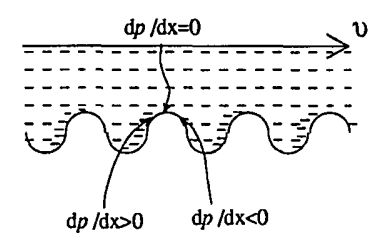

Bearing in Float Polishing

(b)
Fig. 3. (a) Inclined-slipper bearing; (b) float-polishing bearing. Pressure gradients near the peaks of grooves are sufficient to support a load. 


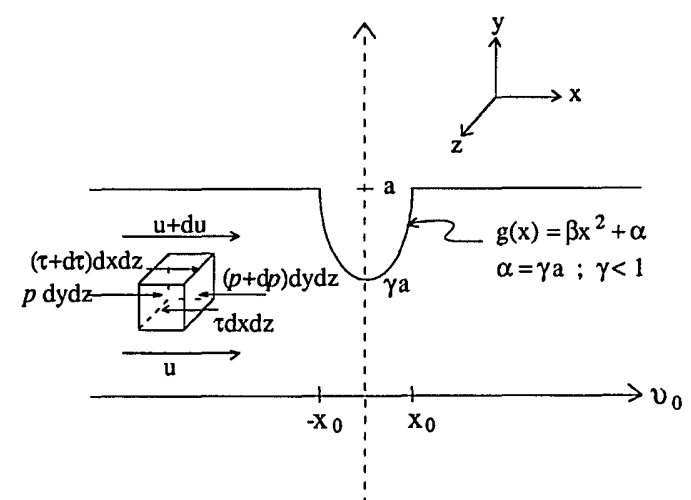

Fig. 4. In this simplified one-dimensional model the pressure gradients near a quadratic boss are calculated to investigate the feasibility of float polishing. $g(x)$ is the groove function, $\alpha$ is the groove height, and $v_{0}$ is the velocity of the moving plane. The frictional forces and pressure on a differential element are as shown.

Making the substitutions for $\tau$ and rearranging we obtain

$$
\frac{\mathrm{d} p}{\mathrm{~d} x}=\frac{\mathrm{d}}{\mathrm{d} y}\left(\eta \frac{\mathrm{d} \nu}{\mathrm{d} y}\right)
$$

and a double integration in $y$ gives

$$
\frac{y^{2}}{2} \frac{\mathrm{d} p}{\mathrm{~d} x}=\eta v+c_{1} y+c_{2} .
$$

The boundary conditions are that the lamina at the upper surface is stationary, and the lamina at the lower planar surface has velocity $\nu_{0}$. This implies that

$$
c_{1}=\left[\frac{g(x)}{2} \frac{\mathrm{d} p}{\mathrm{~d} x}+\frac{\eta v_{0}}{g(x)}\right]
$$

and $c_{2}=-\eta v_{0}$, where $g(x)=\beta x^{2}+\alpha, \alpha=\gamma a, \gamma<1$ is the groove function, and $a$ is the separation between the planes. Explicitly

$$
\frac{y^{2}}{2} \frac{\mathrm{d} p}{\mathrm{~d} x}=\eta\left(\nu-v_{0}\right)+y\left[\frac{g(x)}{2} \frac{\mathrm{d} p}{\mathrm{~d} x}+\frac{\eta v_{0}}{g(x)}\right],
$$

and if a dimension $b$ is assumed in the $z$ direction, neglecting any edge effects, the rate of flow $q_{0}$ can be calculated as follows:

$$
q_{0}=\int_{0}^{g(x)} \mathrm{d} y \int_{0}^{b} \mathrm{~d} z \nu=b\left[-\frac{\mathrm{d} p}{\mathrm{~d} x} \frac{g^{3}(x)}{12 \eta}+\frac{\nu_{0} g(x)}{2}\right] .
$$

The equation is rearranged for the pressure gradient

$$
\frac{\mathrm{d} p}{\mathrm{~d} x}=12 \eta\left[\frac{v_{0}}{2 g^{2}(x)}-\frac{q_{0}}{b g^{3}(x)}\right]
$$

and is expressed explicitly as

$$
\frac{\mathrm{d} p}{\mathrm{~d} x}=12 \eta\left[\frac{\nu_{0}}{2\left(\beta x^{2}+\alpha\right)^{2}}-\frac{q_{0}}{b\left(\beta x^{2}+\alpha\right)^{3}}\right] .
$$

The above equation can be integrated to give

$$
\begin{aligned}
p(x) \approx & 12 \eta\left\{\frac{\nu_{0} x}{4 \alpha}\left[\frac{1}{\left(\beta x^{2}+\alpha\right)}+\frac{1}{\alpha}\right]-\frac{q_{0} x}{2 b \alpha}\right. \\
& \left.\times\left[\frac{1}{2\left(\beta x^{2}+\alpha\right)^{2}}+\frac{3}{4 \alpha\left(\beta x^{2}+\alpha\right)}+\frac{3}{4 \alpha^{2}}\right]\right\}+C,
\end{aligned}
$$

where $C$ is a constant of integration and the approximation

$$
\tan ^{-1}\left(\frac{b x}{\sqrt{\beta \alpha}}\right) \approx\left(\frac{b x}{\sqrt{\beta \alpha}}\right)
$$

is used. To evaluate $C$, let $x=0$, where $p=p_{0}$, which implies that $C=p_{0} . \quad q_{0}$ and $\nu_{0}$ are related quantities and one can determine their relationship by noting that at $x=-x_{0}$, again $p=p_{0}$ and $\left(\beta x_{0}{ }^{2}+\alpha\right)=$ $a$. After some algebra we obtain

$$
q_{0}=2 \nu_{0} a b \frac{\gamma(\gamma+1)}{\left(2 \gamma^{2}+3 \gamma+3\right)},
$$

and if $\gamma=0.5$, then $q_{0}=0.3 \nu_{0} a b$. We can take advantage of the fact that the gradient for the quadratic groove function would be consistently positive for $x<0$ and consistently negative for $x>0$. In particular, if we prove that in some neighborhood in the $x<0$ range that $p(x)>p_{0}$ then it is true for all $x<0$. Substituting the value for $q_{0}$ in Eq. (8) and with $x \sim 0$ we get

$$
p \approx p_{0}-1.2 \eta \frac{\nu_{0} x}{\alpha^{2}},
$$

and, since $x<0, p>p_{0}$. One can calculate the maximum sustainable load by integrating the pressure over the area of the sample.

One can obtain the solution for a grooved profile by modifying $g(x)$. For a groove period $c$ such that $c>$ $\left|x_{0}\right|$,

$$
g(x)=\sum_{n=-\infty}^{\infty} \beta(x-n c)^{2}+\alpha,
$$

or for multiple periods $c$ and $c^{*}$, amplitudes $\alpha$ and $\alpha^{*}$, shapes $\beta$ and $\beta^{*}$,

$$
g(x)=\sum_{n=-\infty}^{\infty} \beta(x-n c)^{2}+\alpha+\sum_{m=-\infty}^{\infty} \beta^{*}\left(x-m c^{*}\right)^{2}+\alpha^{*} .
$$

These expressions can be used in Eq. (7) for the pressure gradient. The pressure given by Eq. (10) with appropriate modifications exists near each crest.

\section{Fabrication Procedure for Float-Polished Quartz}

The two main points made in the above sections are that laminar fluid flow is essential for floating polish- 
ing and that laminar flow of the slurry across the surface results in an ultrafine polishing action. In earlier research, float-polished quartz samples fractured at stress-failure thresholds close to the value of the bulk material, perhaps indicating that minimal subsurface damage was present. ${ }^{6}$ In order to verify the above hypothesis, we analyzed the surfaces of float-polished quartz samples by several techniques to quantify the surface roughness and the presence of subsurface damage resulting from the polishing process.

Natural quartz samples, $25.4 \mathrm{~mm}$ in diameter, were fixed with wax in a hexagonal pattern on a base weighing $2000 \mathrm{~g}$. The samples were lapped on a diamond-turned $\mathrm{Cu}$ lap (see Fig. 1) with a $2 \%$ (by weight) $\mathrm{TiO}_{2}$-deionized $\mathrm{H}_{2} \mathrm{O}$ slurry. The rotational rates of the sample and the lap were set at $55 \mathrm{rpm}$. The average removal rate in this procedure was close to $1 \mu \mathrm{m} / \mathrm{h}$, as measured by a digital micrometer at $4-\mathrm{h}$ intervals. Approximately $10 \mu \mathrm{m}$ was removed from one face and $100 \mu \mathrm{m}$ from the opposite face of each sample. $10-\mu \mathrm{m}$ removal was chosen as a relative marker for comparison with the surface roughness and the subsurface damage level at $100-\mu \mathrm{m}$ removal. The samples were then lapped with a slurry of the same composition as above on a Sn lap for $4 \mathrm{~h}$ to remove an additional 1-2 $\mu \mathrm{m}$ of material. Since Sn is a softer lap material the removal rate is slower than that measured with quartz. This procedure may also reduce the damage layer introduced by the previous process. It is difficult to qualify whether these processes are abrasive or if float polishing does occur. The $\mathrm{TiO}_{2}$ particle size, at less than $100 \mathrm{~nm}$, is well below the fluid layer thickness of $1 \mu \mathrm{m}$. It was observed that the consistency of the $\mathrm{TiO}_{2}$ slurry changed as the lapping progressed. In the first hour of the process, the fresh slurry appeared thick. Later, because of particle breakdown and settling, the slurry appeared less viscous. It is possible that the lapping action converts to a polishing action as the slurry changes.

The samples were final float polished on a remachined $\mathrm{Sn}$ lap with a $2 \%$ fumed $\mathrm{SiO}_{2}$-deionized $\mathrm{H}_{2} \mathrm{O}$ slurry. The slurry was circulated through a constant temperature bath set at $20^{\circ} \mathrm{C}$ to prevent any lap warpage from occurring because of local temperature changes during the polishing process. The rotational rates of the samples and the lap were set at 55 rpm. At this stage we are confident that float polishing does occur for two reasons. First, the operating conditions determine that laminar fluid flow exists between the sample and the lap, and second the $\mathrm{SiO}_{2}$ particle size at $7 \mathrm{~nm}$ is much smaller than the $1-\mu \mathrm{m}$ fluid-layer thickness. Each face of the sample was polished for $8 \mathrm{~h}$ with this procedure. The removal rate in this procedure was extremely slow and not measurable by standard techniques. We removed the wax residue by soaking the samples in 1,1,1trichloroethane. An enamel paint mark was placed on the edge to distinguish the two sides for the purpose of measurements.

\section{Surface and Subsurface Characterization of the Quartz Samples}

We evaluated the quartz samples for surface and subsurface quality by utilizing the techniques of optical scatterometry, photoacoustic spectroscopy, and atomic force microscopy. Of the three techniques photoacoustic spectroscopy is directly sensitive to the level of subsurface damage. Each of the techniques is described briefly below. The measurements are presented as a comparison between the surface with $10-\mu \mathrm{m}$ removal (surface A), used as a reference in which the subsurface damage from preprocessing may still be present, and the surface with $100-\mu \mathrm{m}$ removal (surface B) in which bulk properties would be observed. A Nomarski micrograph and a Talystep scan of a representative area of a polished surface are shown in Fig. 5.

In optical scatter measurements laser light at $\lambda=$ $632.8 \mathrm{~nm}$ is reflected off a test surface, and the off-specular light intensity is measured by a photomultiplier tube. The spot size in our experimental setup was $110 \mu \mathrm{m}$ at the surface. The intensity is plotted in relation to the measurement angle from the specular component. If the profile of the test surface is assumed to be random, it can be decomposed into Fourier components. ${ }^{7}$ These components vary in spatial frequency, amplitude, and relative phase. The specular reflection is generated by the fundamental harmonic, which is a flat surface. Other harmonics cause scatter in random orientations, and the scattered light field is essentially the Fourier trans-
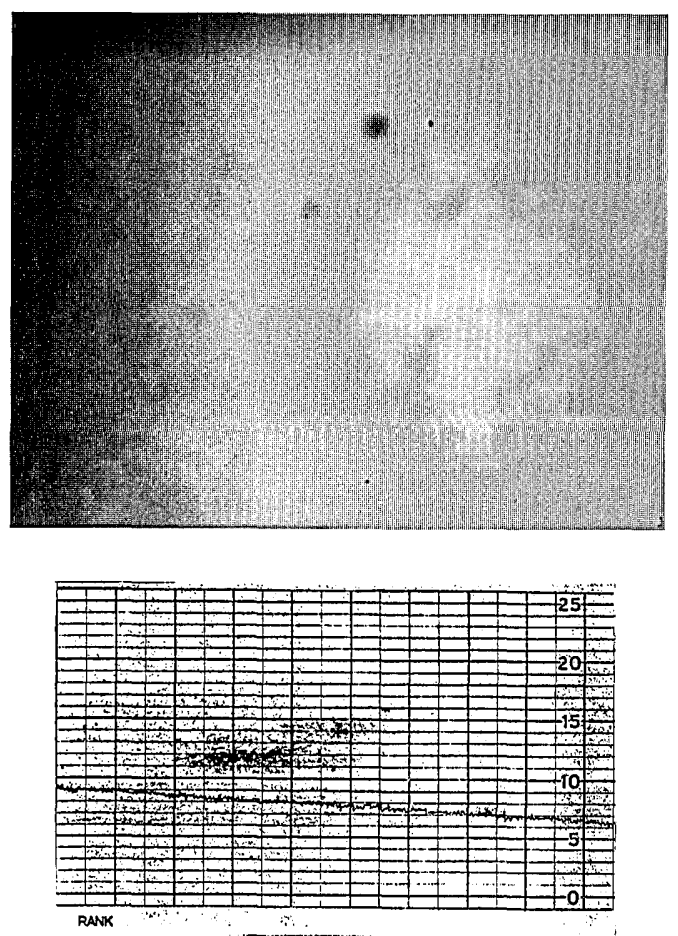

Fig. 5. Nomarski photomicrograph taken at $10 \times$ and a Talystep profile with a vertical resolution of $2 \mathrm{~nm} /$ div and horizontal resolution of $3 \mu \mathrm{m} / \mathrm{div}$ of the surface of a float-polished quartz sample. 
form of the surface; it contains information of the frequency, amplitude, and phase of each harmonic. ${ }^{8}$

Optical scatter measurements of the two polished surfaces of a quartz sample are shown in Fig. 6 . The sharp peak at $120^{\circ}$ in all the graphs corresponds to the region near the specular component. The total scatter from surface B (100 $\mu \mathrm{m}$ of material removed) is approximately ten times lower than that of surface $\mathrm{A}(10 \mu \mathrm{m}$ of material removed). Both surfaces have essentially the same final finish in the polishing process, and under normal conditions one would expect equal amounts of scatter. However, there does appear to be some correlation between the amount of material removed and the scatter amplitude in which the density of subsurface damage could be a variable. The removal of only $10 \mu \mathrm{m}$ is insufficient to eliminate the subsurface damage layer expected from preprocessing (grinding and lapping) of the blanks. It is possible that the subsurface damage layer after the removal of $100 \mu \mathrm{m}$ is shallow and contains fewer scatter sites than the surface with 10 $\mu \mathrm{m}$ of material removed. This shallow subsurface damage layer could be a result of the $\mathrm{TiO}_{2}$ lapping process by which the blanks were prepared.

Photoacoustic spectroscopy is a well-known technique for investigating physical properties of various media. ${ }^{9}$ The photoacoustic technique of surface-

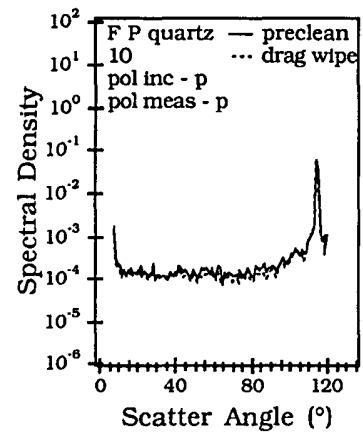

(a)

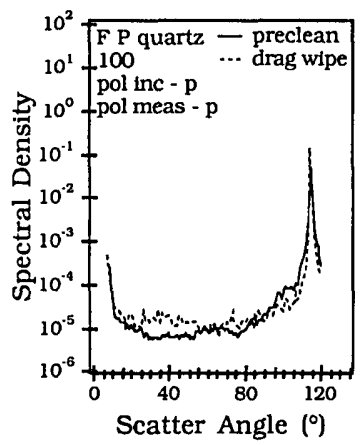

(c)

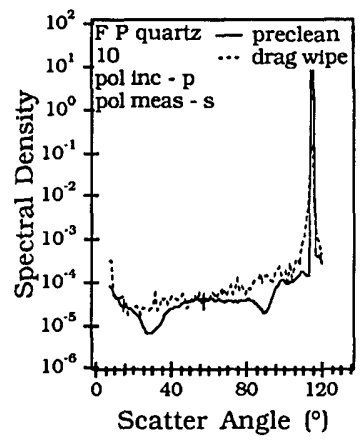

(b)

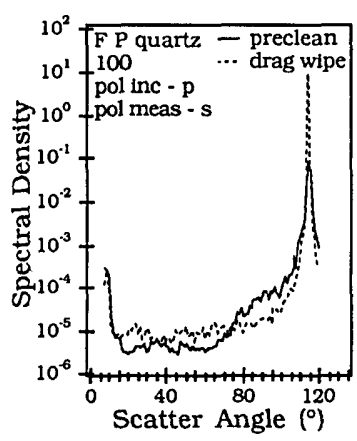

(d)
Fig. 6. Optical scatter measurements with $p$-polarized incident light and both $p$ and $s$ polarizations measured. In both cases the scatter from surface A with only $10 \mu \mathrm{m}$ of material removed (a) and (b) is approximately an order of magnitude higher than that for surface B with $100 \mu \mathrm{m}$ of material removed (c), (d) by float polishing.

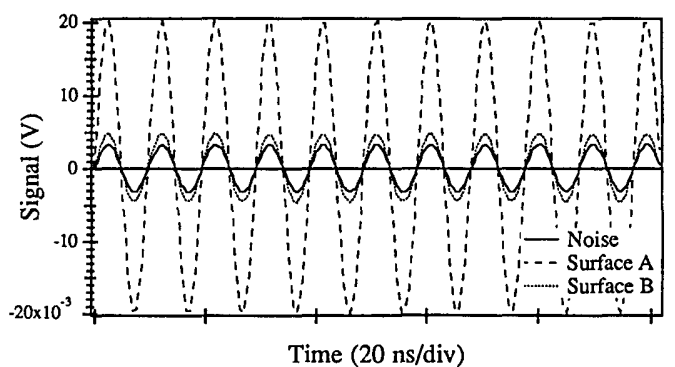

Fig. 7. SAW signals for the two quartz surfaces. The signal for surface $B$ is near the noise floor of the instrument, indicating that the density of subsurface damage is low.

acoustic wave (SAW) detection can be applied to probe surface and subsurface characteristics of optical materials. A system for SAW detection is described in Ref. 10. A pulsed optical beam, having the intensity distribution of a one-dimensional grid pattern, is imaged on the sample. The grid dimension is adjusted to obtain a peak in the SAW signal. The nonradiative fraction of the absorbed radiation causes a local rise in temperature and volume and a corresponding decrease in the local density. This local thermal gradient propagates as a surface wave and is detected by a piezoelectric transducer coupled to the sample. The amplitude of this signal is proportional to the nonradiative fraction, which in turn is proportional to the amount of subsurface damage in the sample.

SAW measurements of the two sides of a floatpolished quartz sample are shown in Fig. 7. There is a definite difference in the amplitudes of the SAW signal between the two polished faces, verifying the hypothesis that the two surfaces have very distinct levels of subsurface damage. Surface A appears to have a deeper region of subsurface damage in which more radiation is absorbed, giving a peak-to-peak SAW amplitude of $40 \mathrm{mV}$ (noise level is less than 10 $\mathrm{mV}$ ). In contrast surface B appears to have a much shallower region of subsurface damage, giving a SAW amplitude of only $10 \mathrm{mV}$, close to the noise floor of the instrument. While some subsurface damage may yet exist within the $100-\mu \mathrm{m}$ face, it could not be readily identified from the SAW signal. Once again this residual subsurface damage layer could be a result of the processing step utilizing a $\mathrm{TiO}_{2}$ slurry.

The atomic force microscope (AFM) is an instrument that is capable of high-resolution profilometry of surfaces. ${ }^{11,12}$ It is similar to a conventional profilometer (e.g., Talystep and Alphastep) in that it produces subnanometer resolution topographic im-

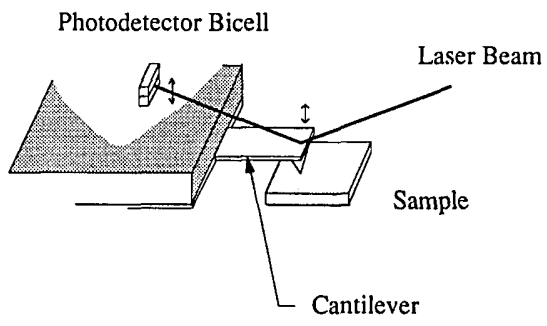

Fig. 8. Optical-lever AFM. 


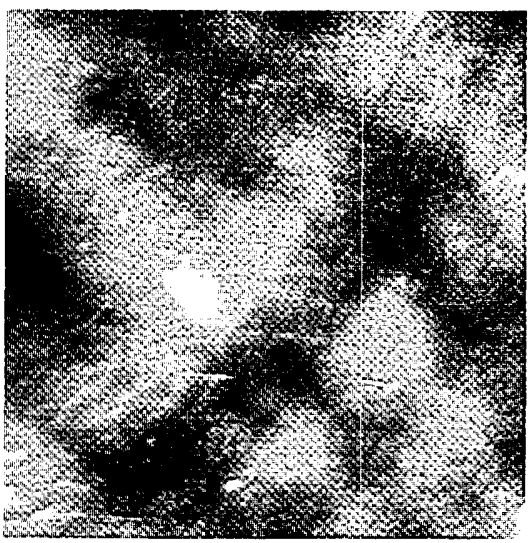

(a)

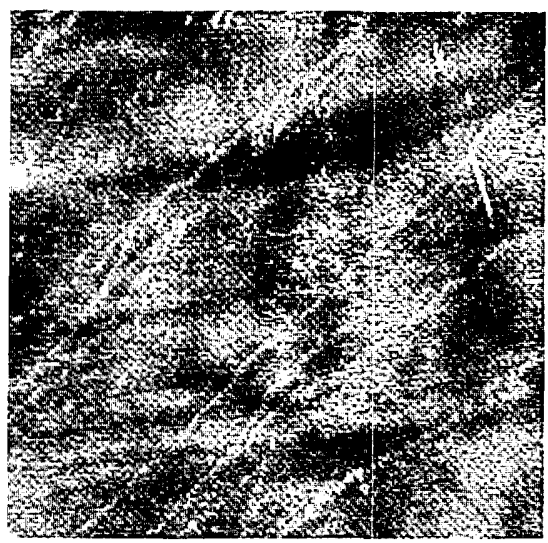

(b)

Fig. 9. A $1.6 \mu \mathrm{m} \times 1.6 \mu \mathrm{m}$ AFM image of (a) float-polished quartz surface $A$ and $(b)$ float-polished quartz surface $B$.

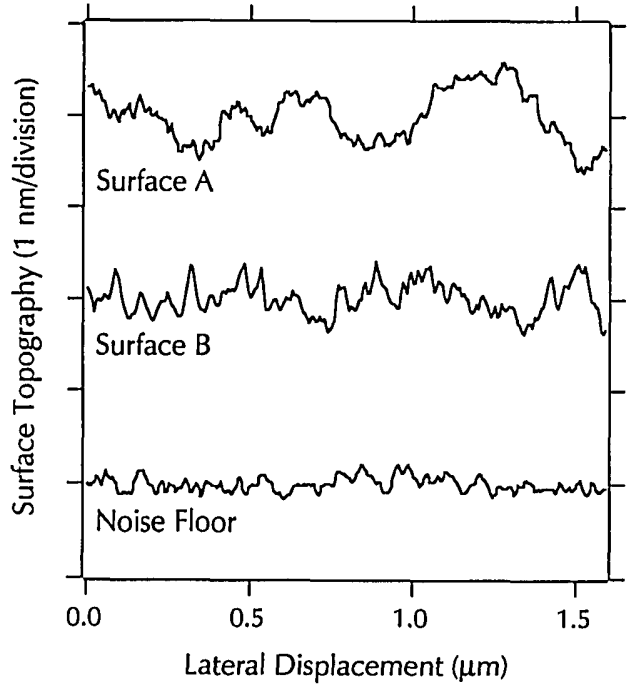

Fig. 10. AFM topographic images of float-polished quartz. Surface A (10- $\mu \mathrm{m}$ material removed) had a roughness of less than $0.5-\mathrm{nm} \mathrm{rms}$, while the roughness for surface $B(100-\mu \mathrm{m}$ material removed) was approximately twice as better at $0.2-\mathrm{nm} \mathrm{rms}$. The noise floor of the instrument is approximately $0.1-\mathrm{nm} \mathrm{rms}$ and is also shown.

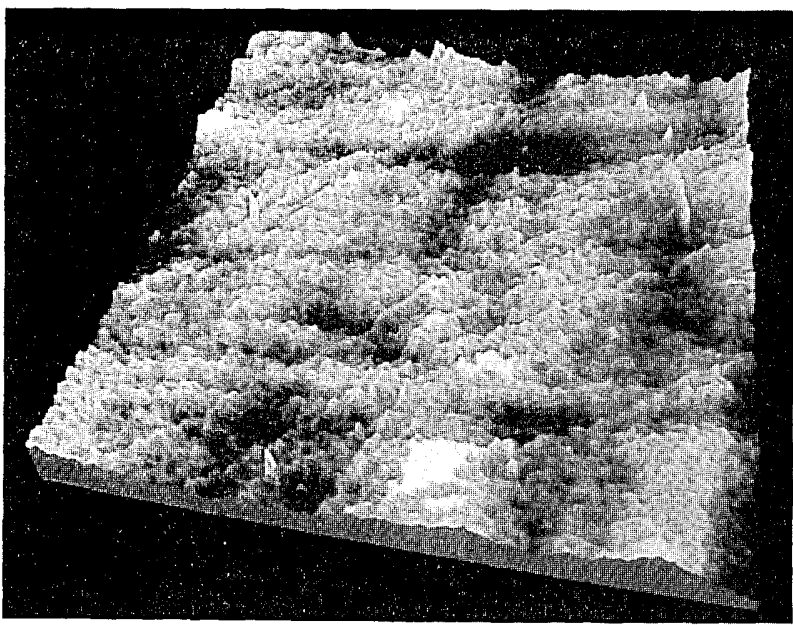

Fig. 11. Three-dimensional atomic force micrograph of surface B.

ages by scanning a tip attached to a cantilever spring over the sample. In an AFM the displacement of the tip is measured with an optical layer. The optical lever consists of a laser source, a reflectorized cantilever, and a two-segment photodetector (Fig. 8). The force exerted on the sample is held constant by raising or lowering the cantilever with a feedback loop as the sample is scanned. The float-polished quartz samples were imaged with an AFM constructed in house. Commercially available AFM cantilevers with specially sharpened tips (supertips) were used. The tip size was less than $5 \mathrm{~nm}$. The force exerted on the sample was in the neighborhood of $10^{-8} \mathrm{~N}$, and the noise level was approximately $0.1-\mathrm{nm} \mathrm{rms}$. The images contain $250 \times 250$ data points scanned at a rate of 5 lines / $\mathrm{s}$.

Tests on atomically flat, highly oriented pyrolytic graphite have demonstrated that the AFM produces a spurious overall image curvature of approximately $3.5 \mathrm{~nm}$ over a $1-\mu \mathrm{m}$ scan. ${ }^{13}$ Although we removed most of the curvature by subtracting a best-fit paraboloid from each image, some higher-order distortion may yet exist in the images. Atomic force micrographs of the two float-polished faces are shown for comparison in Figs. 9(a) and 9(b), and the corresponding line profiles are shown in Fig. 10. Surface A had $0.5-\mathrm{nm} \mathrm{rms}$ surface roughness and frequently exhibited shallow scratch marks, originating possibly from preprocessing steps. Surface B had approximately $0.2-\mathrm{nm} \mathrm{rms}$ surface roughness and generally had narrow line features that may be residual subsurface damage marks or grain boundaries. A three-dimensional image of surface B is presented in Fig. 11. Occasional granularity is seen in both images, which we believe to be artifacts caused by damaged or dull tips. ${ }^{14}$

\section{Conclusions}

Float polishing as a fluid mechanical flow operates in the laminar regime. The one-dimensional model we presented shows that laminar flow of the slurry between the sample and the lap results in pressure gradients in the grooves. The pressure gradients 
cause the sample to float above the lap on a layer of slurry. Laminar flow in the system also results in a high-quality polish since the substrate is isolated from the lap. Quartz samples for use in high-stress environments were polished in a three-stage process with $\mathrm{TiO}_{2}$ and $\mathrm{SiO}_{2}$ slurries and have a supersmooth $0.2-\mathrm{nm}$ rms finish. By optical scatterometry and photoacoustic spectroscopy measurements of the polished surfaces, it has been shown that the subsurface damage level for a surface with $100-\mu \mathrm{m}$ removal was low in comparison with a surface with $10-\mu \mathrm{m}$ removal. At $10 \mu \mathrm{m}$ the removal is insufficient to eliminate the subsurface damage layer preprocessing.

We acknowledge the assistance of Scott Stowell and David Reicher in the fabrication and measurements reported in this paper. This work was supported by the Air Force Office of Scientific Research under the Generated Surfaces and Thin Film Coating Development Task at Phillips Laboratory, Kirtland Air Force Base, Albuquerque, N.M.

\section{References and Notes}

1. Y. Namba and H. Tsuwa, "Mechanism and some applications of ultra-fine finishing," Ann. CIRP 27, 511-516 (1978).

2. Y. Namba, Department of Mechanical Engineering, Chubu University, Kasugai, Aichi 487, Japan (personal communication, 1989).

3. See Science of Optical Finishing, Vol. 9 of OSA 1990 Technical Digest Series (Optical Society of America, Washington, D.C., 1990).
4. H. Schlichting, Boundary-Layer Theory, 7th ed. (McGraw-Hill, New York, 1979), Chap. 2.

5. B. S. Bassey, Mechanics of Fluids, 4th ed. (Van NostrandReinhold, New York, 1979), Chaps. 5 and 6.

6. S. F. Soares, K. C. Jungling, and W. K. Stowell, "Float polished quartz substrates," in Science of Optical Finishing, Vol. 9 of OSA 1990 Technical Digest Series (Optical Society of America, Washington, D.C., 1990), paper JTuA3.

7. M. S. Longuet-Higgins, "The statistical analysis of a random, moving surface," Philos. Trans. R. Soc. London Ser. A 249, 321-387 (1957).

8. E. L. Church, H. A. Jenkinson, and J. M. Zavada, "Relationship between surface scattering and microtopographic features," Opt. Eng. 18, 125-136 (1979).

9. A. C. Tam, "Applications of photoacoustic sensing techniques," Rev. Mod. Phys. 58, 381-430 (1986).

10. M. Y. A. Raja, D. W. Reicher, S. R. J. Brueck, J. R. McNeil, and D. E. Oates, "High-sensitivity surface-photoacoustic spectroscopy," Opt. Lett. 15, 66-68 (1990).

11. G. Binnig, C. F. Quate, and Ch. Gerber, "Atomic force microscope," Phys. Rev. Lett. 56, 930-933 (1986).

12. S. A. C. Gould, B. Drake, C. B. Prater, A. L. Weisenhorn, S. Manne, H. G. Hansma, P. K. Hansma, J. Massie, M. Longmire, V. Elings, B. D. Northern, B. Mukherjee, C. M. Peterson, W. Stoockonius, T. R. Albrecht, and C. F. Quate, "From atoms to integrated circuit chips, blood cells, and bacteria with the atomic force microscope," J. Vac. Sci. Technol. A 8, 360-373 (1990).

13. D. R. Baselt and J. D. Baldeschwieler, "Lateral forces during atomic force microscopy of graphite in air," J. Vac. Sci. Technol. B 10, 2316-2322 (1991).

14. P. Grutter, W. Zimmermann-Edling, and D. Brodbeck, "Tip artifacts of microfabricated force sensors for atomic force microscopy," Appl. Phys. Lett. 60, 2741-2743 (1992). 